\title{
Observing Black Holes Spin
}

\author{
Christopher S. Reynolds \\ Institute of Astronomy, Cambridge, CB3 OHA
}

\begin{abstract}
The spin of a black hole retains the memory of how the black hole grew, and can be a potent source of energy for powering relativistic jets. To understand the diagnostic power and astrophysical significance of black hole spin, however, we must first devise observational methods for measuring spin. Here, I describe the current state of black hole spin measurements, highlighting the progress made by X-ray astronomers, as well as the current excitement of gravitational wave and radio astronomy based techniques. Today's spin measurements are already constraining models for the growth of supermassive black holes and giving new insights into the dynamics of stellar corecollapse, as well as hinting at the physics of relativistic jet production. Future X-ray, radio, and gravitational wave observatories will transform black hole spin into a precision tool for astrophysics and test fundamental theories of gravity.
\end{abstract}

A black hole is defined by its event horizon, the point of no return inside of which even light is destined to be pulled inwards. Einstein's Theory of General Relativity (GR) tells us that the region interior to the event horizon follows the Las Vegas rule; what happens in the black hole interior stays in the black hole interior. This causal disconnection has a remarkable consequence; outside of the event horizon, black holes are nature's simplest objects, defined solely by their electrical charge (which is neutralized to zero in realistic astrophysical settings), mass, and angular momentum [1, 2].

The angular momentum, or spin, of a black hole has tremendous astrophysical importance. An enormous amount of energy can be stored in the spin and tapped to drive astrophysical processes this is now the standard theoretical model for how the spectacular jets in active galactic nuclei (AGN) are driven [3], with the spin of a central supermassive black hole (SMBH) able to energize powerful jets for hundred of millions of years before becoming exhausted. In many cases, these jets plough into gas within their host galaxies, depositing their energy and stifling star formation [4], making black hole spin integral to the story of galaxy formation. Spin also provides a fossil record of how the black hole formed. For example, the formation of SMBHs in the early Universe is still deeply mysterious, and whether the population of such black holes is rapidly spinning or not can differentiate scenarios where they form from coherent disk-accretion or the chaotic merger of smaller black holes [5].

For these reasons, there has been significant interest in developing methods for measuring the spin of astrophysical black holes [6]. In this Review, I will survey the current state and future promise of black hole spin measurements. For much of the past 20 years, quantitative measures of spin have been the domain of X-ray astronomy, and these techniques continue to be refined as the quality of the data improves. With the recent advent of gravitational wave astronomy, we now have a completely new and complementary window on spinning black holes. Furthermore, we stand on the threshold of another major breakthrough, the direct imaging of the shadow of the event horizon by global mm-band Very Long Baseline Interferometry, aka, the Event Horizon Telescope (EHT). We are truly entering a golden age for the study of black hole physics and black hole spin. 


\section{What is black hole spin?}

Before embarking on a discussion of spin measurements, we must address a fundamental question given the perfect nature of a black hole (i.e. the complete absence of "surface" features), what exactly does it mean for a black hole to rotate?

Assuming that gravity is described by Einstein's General Theory of Relativity (GR), the mathematical description of an isolated and uncharged spinning black hole was found by Roy Kerr in the early 1960s [7]. The Kerr solution shows that the spacetime outside of a spinning black hole rotates around the black hole like a vortex. This rotating spacetime will tend to drag nearby matter, and even light, into rotation around the black hole. This effect, known as frame-dragging, is weak far from the black hole and matter is able to resist (if, for example, it has a rocket motor attached!). Sufficiently close to the spinning black hole, within a location known as the static limit, the frame-dragging becomes irresistible and all matter and light is forced to rotate around the black hole.

This zone of irresistible rotation is important for understanding the energetics of a spinning black hole. In a classic thought experiment, Roger Penrose [8] showed that the energy of rotation resides within this region and can in principle be extracted. This gives the name to this region - the ergosphere (derived from the Ancient Greek ergon meaning "work"). While the original Penrose process may be hard to realise in nature, Roger Blandford and Roman Znajek showed that magnetic fields can similarly extract rotational energy from the ergosphere [3]. Magnetic spin-extraction is the leading theoretical model for the driving of relativistic jets from black hole systems.

To be more quantitative, we consider a black hole with mass $M$ and angular momentum $J$. We can define the unitless "spin parameter" by $a=c J / G M^{2}$ where $c$ is the speed of light and $G$ is Newton's constant of Gravitation. The Kerr solution tells us that the structure of the spacetime around a spinning black hole depends only on $M$ and $a$. As well as greatly simplifying any GR treatments of black hole astrophysics, this provides a route to observational explorations of gravity theories beyond GR - once the mass and spin of an astrophysical black hole has been measured, we can in principle search for deviations of the inferred gravitational field (including any gravitational radiation) from the predictions of GR.

If one were to spin a planet or a star too quickly, it would fly apart as the centrifugal forces overwhelm the gravity that binds the object together. There is an equivalent situation for a black hole. The Kerr solution shows that, if $|a|>1$, there is no longer an event horizon. GR would then predict a naked spacetime singularity, an outcome that is abhorrant to physical law and the notion of predictability and thus forbidden by the Cosmic Censorship Hypothesis. Of course, it is of great interest to physicists to test whether nature respects this Kerr limit [9].

\section{Accretion disks, X-rays and black hole spin}

Until recently, spin measurements were only possible for the accreting SMBHs found in AGN or the accreting stellar mass black holes in X-ray binaries. In both cases, the accreting gas forms a rotating flattened disk — an accretion disk — whose structure is affected by the frame-dragging associated with the black hole's spin. The key challenge is to understand the influence of frame-dragging on accretion disk structure and compare these expectations to data from real black holes.

In general, accretion disks are complex systems and we have yet to fully understand the influence of black hole spin on their structure. However, if the accretion rate is in a goldilocks zone - not too high and not too low — the accreting gas can efficiently radiate the liberated energy as it flows inwards leading to a geometrically-thin, pancake-like disk [11]. Far from the black hole, where the gravitational field is well approximated by Newton's law of gravity, the gas in such disks follows stable circular orbits, with a small inward drift producing the actual accretion. As the gas nears the black 


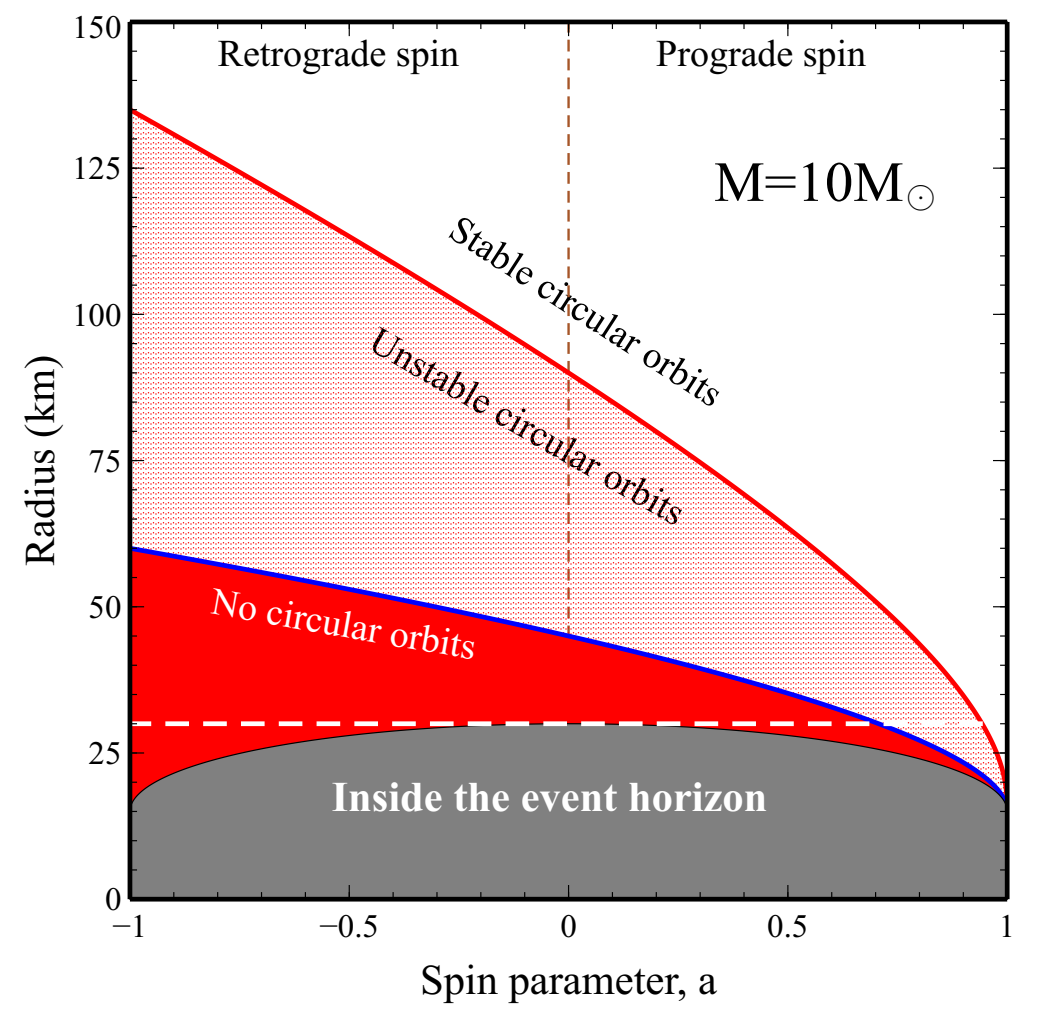

Figure 1: Location of some special orbits in the equatorial plane of a Kerr black hole as a function of spin parameter.. Shown here is the innermost stable circular orbit (red line), photon circular orbit (blue line), static limit (dashed white line), and event horizon (bounding the grey shade). Positive/negative spin parameter corresponds to spin that is prograde/retrograde, respectively, relative to the orbiting matter (or photons). The vertical dashed red line separates the prograde and retrograde cases. Circular orbits are stable outside of the innermost stable orbit but become unstable inside of this radius (region denoted by light red shading). Circular orbits do not exist interior to the photon circular orbit (region denoted by solid red shading). For concreteness, a 10 solar mass black hole is assumed. Radii for other masses can be obtained using linear proportionality. Figure follows [10].

hole, relativistic effects become important, de-stabilising circular orbits and causing the accreting gas to undergo a plunging spiral into the black hole event horizon [12, 13]. The location of this transition, the innermost stable circular orbit (ISCO), is a basic property of the black hole's gravitational field and depends on the spin [10]. If the black hole and the accretion disk are rotating in the same sense, framedragging effects can stabilize otherwise unstable gas motions, resulting in the ISCO being progressively closer to the black hole as we consider higher spins (Figure 1).

At least in these geometrically-thin accretion disks, the ISCO effectively acts as the inner edge to the accretion disk (Figure 2) and so imprints itself on the emergent electromagnetic radiation, especially the X-ray emission from the inner disk [12, 13]. This provides the route to measuring spin from X-ray observations.

The technique employed most extensively to date for measuring spins is based on gravitational redshifts of atomic features in the X-ray spectrum. In both AGN and (many) accreting stellar mass black holes in X-ray binaries, we see powerful high-energy X-ray emissions from a corona of extremely 


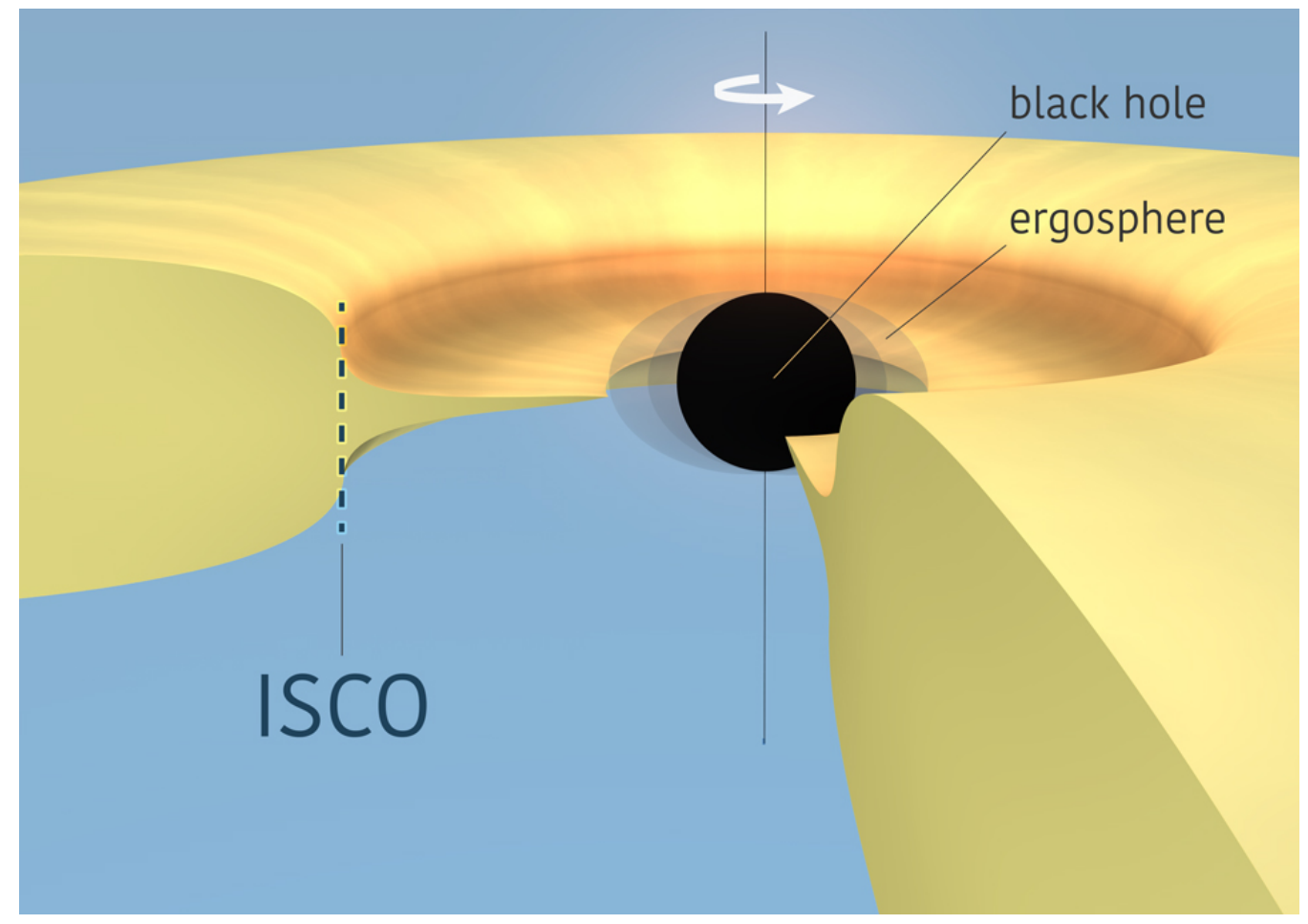

Figure 2: Cartoon of the inner regions of a geometrically-thin accretion disk showing the transition in disk structure at the innermost stable circular orbit (ISCO). Within the ISCO, the matter is too highly ionized to produce atomic spectral features and the emission processes are non-thermal. Figure courtesy of Amanda Smith (Institute of Astronomy, Cambridge).

hot $\left(T \sim 10^{9} \mathrm{~K}\right)$ electron/positrons pairs above the accretion disk [14]. The accretion disk, which is much cooler, is strongly irradiated by these X-rays causing chemical elements in the surface layers to become photo-excited and produce fluorescent emission lines at very well-defined X-ray frequencies [15; this process is referred to somewhat inappropriately as X-ray reflection. Given that we are always viewing the accretion disk at some angle away from face-on, the observed emission lines from the disk are shifted in energy by (i) the normal Doppler effect (as the material on its orbit approaches us and then recedes again leading to blueshifts and redshifts respectively), (ii) the time-dilation of Special Relativity (moving clocks run slowly leading to redshifting), and (iii) the gravitational redshifting of General Relativity [16]. The emission lines become highly broadened and asymmetric, with prominent blueshifted peaks and long redshifted tails. The spin of the black hole is encoded in these line profiles - as one considers black holes of progressive higher spin, the ISCO moves closer to the event horizon, the gravitational redshift of X-rays reflected from the ISCO increases, and the extend of the redshifted tail of the iron line grows. [17, 18]. These are subtle signatures [19]. X-ray reflection from more distant gaseous structures as well as X-ray absorption from material in the Milky Way, the host galaxy of the AGN, and any accretion disk wind must be carefully modeled and accounted for before the subtle effects of spin can be teased from the data. In particular, the degeneracy of spin signatures with those of accretion disk winds has sparked lively debate [20, 21, 22. Recent work has also explored systematic errors in spin measurements that result from the finite thickness of the accretion disk [23], finding them to become increasingly relevant as one considers black holes with higher accretion rates.

Broadened X-ray reflection has been observed and probed for spin signatures in both AGN and 
(a)

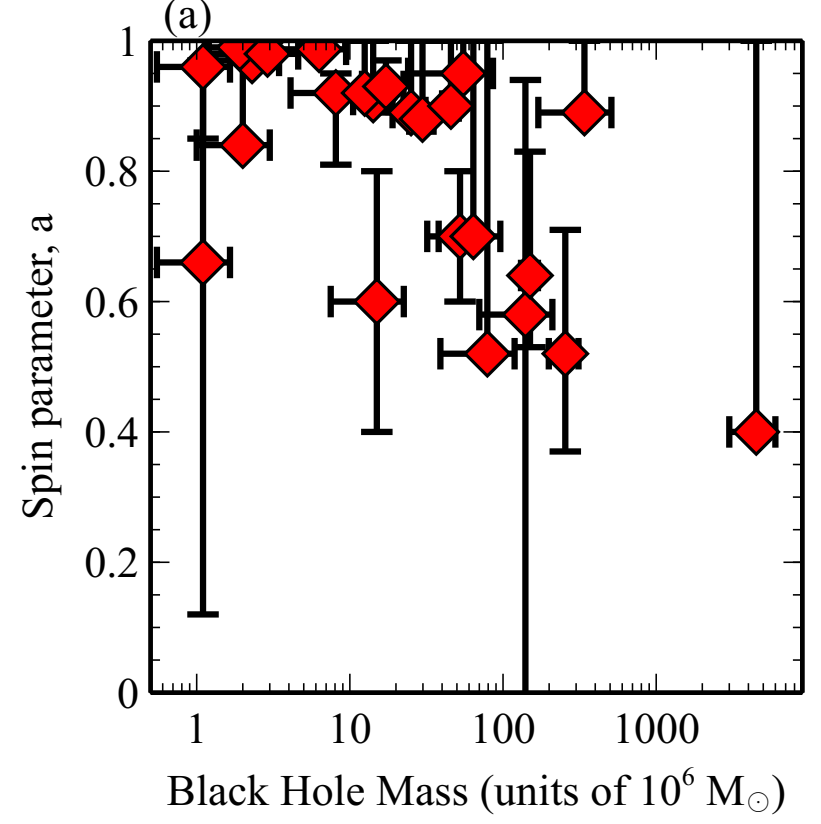

(b)

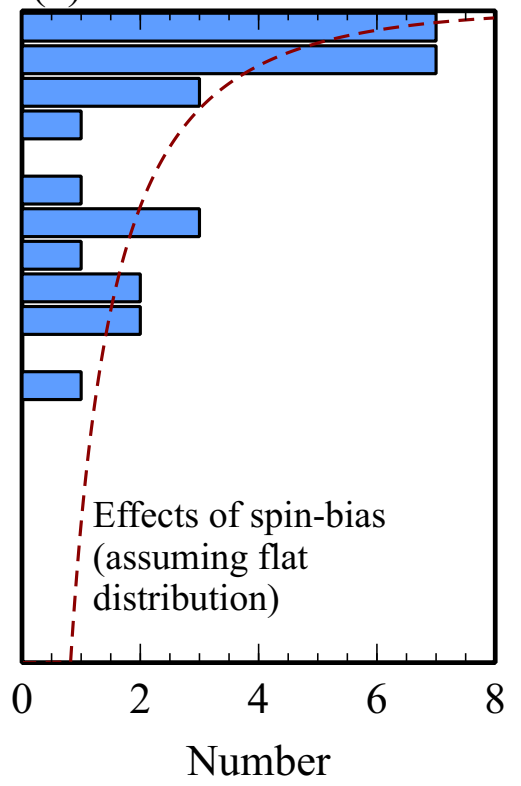

Figure 3: Results on the spins of supermassive black holes in active galactic nuclei using the X-ray reflection method. Panel-a shows the spins and masses of 22 black holes with robust results. Following the conventions in the relevant primary literature, we show $90 \%$ confidence error bars on black hole spin but $1 \sigma$ errors on black hole mass. Panel-b shows the distribution of these spins along with the expectations based on a simple efficiency-based selection-bias assuming an underlying population of black holes that has equal numbers as a function of spin parameter. Figure based on [34, updated with results from [30, 31, 32, 29. Note that four objects (IRAS00521-7054, SwiftJ0501.932.39, RBS1124, and $1 \mathrm{H} 0323+342$ ) have poor SMBH mass constraints; these have been included in the spin distribution (right) but not the mass-spin plane.

X-ray binaries for over 20 years [16, 24, 25, 26, 18, 27, 28, 22, 29, 30, 31, 32. In the realm of AGN, there are robust spins for over two dozen SMBHs [33, 34]. As shown in Figure 3a, we find that below about 30 million solar masses, the vast majority of SMBHs examined seem to be rapidly spinning $(a \gtrsim 0.9)$. For more massive black holes, however, we appear to pick up a population of more slowly spinning objects $(a \sim 0.5-0.7)$. As discussed later, this may hint at different formation modes in the two mass regimes.

Interestingly, there may be selection biases which favour finding high-spin black holes in AGN. Fundamentally, AGN are chosen for study because they are bright (or, more technically, they are usually drawn from flux-limited samples). For a given mass flow rate onto the black hole, the accretion disk around a more rapidly spinning hole will release more energy and hence be over-represented in any sample based on brightness thresholds [34, 35]. A full understanding of these selection biases is still ongoing work but, in a simple treatment, the observed spin distribution (summed over all masses) is statistically consistent with an underlying population that has equal numbers of black holes with spins between $a \approx 0.4$ and the extreme limit $(a=1)$, as shown in Figure $3 \mathrm{~b}$.

$\mathrm{X}$-ray reflection based spin measurements do not require knowledge of either the mass of the black hole or its distance from us since the gravitational redshift of the ISCO depends only on the spin parameter. This means that it can be equally applied to AGN (where the black hole mass is often 
poorly determined) and the stellar-mass black holes in X-ray binaries (where the distance can be highly uncertain [36]). To date, 15 stellar-mass black holes (14 within our Galaxy and one in the Large Magellanic Cloud) have reflection based spin measurements, revealing a wide range of spins from rather slowly rotating $(a=0.3-0.4)$ to very rapidly spinning $(a>0.95)$ objects [37, 33]. This observed diversity makes sense since we expect the selection biases affecting AGN to be absent from these stellar-mass results — we essentially know of and have studied every stellar-mass black hole in our Galaxy that has undergone an outburst in the past 25 years, so we have a "fair-sample" of these objects.

The second major technique that has been extensively used to measure black hole spin employs the temperature of the accretion disk to diagnose the location of the ISCO. Specialising again to geometrically-thin accretion disks, we expect much of the liberated energy to be radiated as thermal emission (with an approximate blackbody form) from the disk surface, a disk around a rapidly spinning black hole possessing higher temperatures (and brighter emission) than a similar disk around a nonspinning black hole [38, 39]. Building models of the thermal spectrum that take into account the detailed structure of the accretion disk as well as the influence of the Doppler effect and gravitational redshifting, we can compare to observations and determine spin. Unlike in the gravitational-redshift based methods, we must also have knowledge of the black hole mass, inclination of the accretion disk, and distance from the Earth in order to build these models.

This "thermal continuum fitting" technique is particularly effective for accreting stellar-mass black holes in X-ray binary star systems. In these systems, the inner accretion disks are hot $\left(T \sim 10^{7} \mathrm{~K}\right)$, placing the bulk of their emission in the low-energy X-ray part of the spectrum where it can penetrate the dust and gas of our Galaxy, and where we have sensitive detectors on space-based observatories. The fact that the gas in the surface layers of these hot accretion disks is highly ionized leads to only small distortions from a blackbody spectrum, making the spectral models easier to calculate and more robust [40]. We can also use classical techniques in optical astronomy to extract the black hole mass and accretion disk inclination from analyses of the orbit of the companion star [41. Finally, stellarmass black holes cycle through different states and so we can wait for them to enter a state where the thermal emission is completely dominant over the high-energy X-ray emitting corona [42], validating the assumptions underlying the continuum fitting technique.

To date, 10 stellar-mass black holes ( 7 in our Galaxy, two in the Large Magellanic Cloud, and one in the dwarf galaxy M33) have thermal continuum fitting based spin measurements [43, 44, 45, 46]. Six of these objects have both thermal continuum and X-ray reflection based spin measures, allowing us to examine consistency of results. Only for one object, 4U1543-47, does a significant discrepancy exist with $a=0.3 \pm 0.1$ from X-ray reflection [37] and $a=0.8 \pm 0.1$ from thermal continuum fitting [43. However, both of these measurements were made with old data from the Rossi X-ray Timing Explorer (RXTE) which had limited low-energy X-ray sensitivity and limited spectral resolution. So it is not surprising if one or both of these measurements is compromised by systematic errors. With new soft X-ray data coming from the Neutron star Interior Composition ExploreR (NICER) and greatly improved distance estimates from Gaia [36, we expect a new surge of spin results based on continuum-fitting over the next few years.

Application of the thermal continuum fitting method to AGN is more challenging. The black hole masses are often highly uncertain, and the inner accretion disks are relatively cool $\left(T \sim 10^{5} \mathrm{~K}\right)$ with the bulk of the thermal emission in the far-or-extreme ultraviolet region of the spectrum. This part of the spectrum is strongly absorbed by the dust and gas in our Galaxy, making it hard to even see this radiation from an AGN. Furthermore, atomic processes in the surface layers of these cool disks also distorts the thermal spectrum away from blackbody form, making it more challening to calculate robust models [47]. Indeed, the surprising degree of optical/UV variability displayed by AGN has called into question whether a thermally-emitting standard accretion disk is even the right 
basic description for the optical/UV part of the spectrum [48. Still, there have been several studies of SMBH spin using variants of thermal continuum fitting [49, 50, 51, 52]. Encouragingly, for two objects that have well constrained masses as well as both X-ray reflection and thermal continuum spin measurements, the results are in good agreement [51].

\section{Gravitational wave astronomy and black hole spin}

On the 15th September 2015, the advanced Laser Interferometer Gravitational-Wave Observatory (aLIGO) detected the gravitational waves as two orbiting 30 solar mass black holes underwent their final merger in a galaxy more than a billion light years away [53] — this initiated the era of gravitational wave astronomy and has opened a new window on black hole spin.

There are three distinct stages to the merger of a binary black hole system. During the inspiral stage, the two black holes orbit around their common centre of mass, slowly losing energy and angular momentum via gravitational waves. The black holes speed up as the orbit decays leading to a gravitational wave train that becomes stronger and higher frequency as time goes on. Eventually, the black holes get close enough that they pass the binary equivalent of the ISCO. They plunge together in a merger stage that produces extremely powerful and complex gravitational wave signals. The result is a single spinning black hole that starts off highly perturbed but quickly settles down to a Kerr black hole by emitting gravitational waves in a stage called ring-down. In principle, the full gravitational wave signal contains information about the spins of the two initial black holes, the orientation of these spins relative to each other and the orbital axis of the binary, and the spin of the final black hole. This is also a "clean" technique in the sense that it is pure gravitational evolution and the inspiral-merger-ringdown wave forms do not depend upon the complex physics of accretion.

There are of course practical challenges to accessing this clean information due to the finite signalto-noise and limited frequency range of real gravitational wave detectors. For the current generation of gravitational wave observatories, the target black holes are in the stellar-mass range and the bulk of the signal is obtained during the inspiral stage. It can be shown using approximate analytic calculations (so-called post-Newtonian theory) that the phasing of the gravitational wave signal during inspiral is influenced by the mass-weighted average of the individual black hole spins projected onto the axis around which the black holes are orbiting — this is called the effective spin, denoted $\chi_{\text {eff }}$ [54]. The components of the individual spins that project into the plane of the orbit cause precession of that plane. This is known as the precession spin [55], denoted $\chi_{\mathrm{p}}$ and is also imprinted onto the gravitational wave form but in more subtle manner.

To date, aLIGO working in concert with the European VIRGO interferometer have high-significance detections of four black hole binary mergers [53, 56, 57, 58]. The first event GW150915 was consistent with merging non-rotating black holes [59] - the effective spin was constrained to be $\chi_{\text {eff }}=-0.04_{-0.16}^{+0.14}$ (with $90 \%$ confidence) meaning that we can rule out rapidly spinning black holes that are both aligned or anti-aligned with the orbital axis. The constraints on the precession spin were weak so we cannot tell whether the black holes are truly rotating slowly, or whether their rapid spins lie in the plane of the orbit. In the second event, GW151226, there was a significant detection of effective spin, showing that at least one of the black holes has a spin parameter greater $a>0.2$ and that this spin is at least partially aligned with the orbital axis [56] (Figure 4). Again, though, no additional information could be gleamed from the precession spin.

We can also examine the spin of the final black hole left once the merger is complete. In principle, this spin is imprinted cleanly on the spectrum of the gravitational waves from ring-down [60, 61]. At current sensitivities, though, the ring-down emission is difficult to detect and characterize. However, this final spin is determined to a large degree by the mass ratio and effective spins of the progenitor binary system. Taking measurements of these quantities from the inspiral signal, computer simulations 


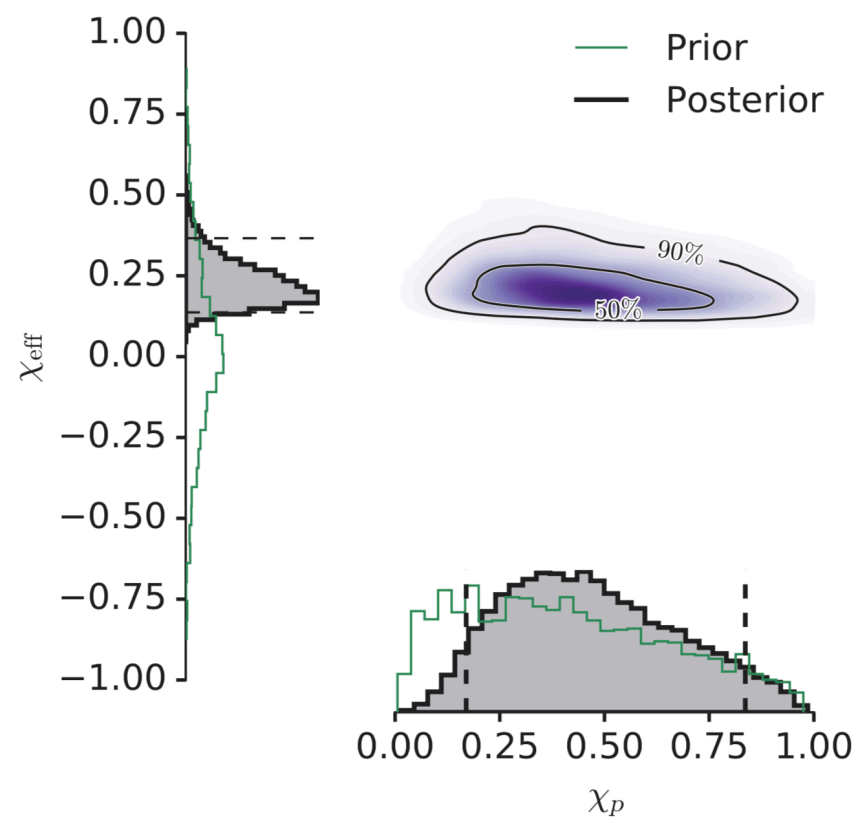

Figure 4: Constraints on black hole spin for GW151226. Shown here are the derived probability distribution (black lines with grey shading) and initially assumed prior probability (green) for the effective spin $\chi_{\text {eff }}$ and precession spin $\chi_{\mathrm{p}}$. The joint 2-dimensional probability distribution on the $\left(\chi_{\mathrm{eff}}, \chi_{\mathrm{p}}\right)$ is shown in purple, along with $50 \%$ and $90 \%$ confidence contours. Figure from [56].

of the full merger process can be used to infer the final spin typically giving values in the range $a=0.5-0.8$.

\section{The spin of the Galactic Centre black hole}

The closest SMBH to us is in the centre of our own Galaxy, about 25,000 light years away weighing in at 4 million solar masses. To date, there is no generally accepted measurement of the spin of this black hole. The accretion rate is extremely low leading to a tenuous and extremely hot accretion disk that is significantly puffed up into a fat donut geometry [62, 63], and such an accretion disk does not produce X-ray reflection features or the thermal blackbody radiation described above. Also, being a single black hole, we do not expect any gravitational waves and, even if there were some unknown companion black hole, the resulting gravitational waves would be of too low frequency for aLIGO. However, the unusual proximity of this black hole offers some unique possibilities for study which are currently being actively developed [64, 65] and may lead to robust measures of the spin of this object in the very near future.

Firstly, we have the opportunity to literally take a picture of this black hole. The event horizon of the Galactic Center black hole will appear to cast a shadow against the glow of the background accreting matter [66] with an apparent size of 50 micro-arcseconds (14 billionths of a degree, or half the size of a football on the Moon viewed from the Earth). Such imaging is possible with the Event Horizon Telescope (EHT), an interferometric experiment between a network of radio telescopes spread over the Earth operating at a wavelength of $1.3 \mathrm{~mm}[67,68]$. The fully operational EHT has conducted observing campaigns on the Galactic Centre in April 2017 and May 2018 and, at the time of writing, the data are still being analysed. As well as conclusively proving the existence of the event horizon 
for the first time, the EHT images of the inner accretion disk promise to be a unique probe of the inclination of the accretion disk, the physical processes that make it glow, and the black hole spin. While the size of the shadow is rather insensitive to the spin, the asymmetry in brightness between the approaching and receding sides of the disk is expected to be strongly spin dependent. Indeed, if the black hole has a particularly extreme spin ( $a$ very close to 1$)$, the image of the inner accretion disk collapses to a vertical line on the approaching side [69]. However, preliminary data from prototype EHT runs suggest that this SMBH has a low spin parameter [70, 71]. There is model dependence to this result, though, caused by time variability of the accretion disk and the foreground distribution of Galactic gas that acts as a time varying scattering screen [72]. We must await multi-epoch data from the full EHT before a robust spin can be derived.

Secondly, the proximity of the Galactic Centre means that we can observe individual stars orbiting close to the black hole. As well as already giving us precision measures of the mass of this black hole [73, 74, 75, it opens the possibility of seeing the stellar orbits precess due to frame-dragging associated with the black hole spin. Such measurements require finding and tracking stars that are within just a few hundred event horizon radii from the black hole [76], a task best done with multiyear near infrared interferometry. This is the main focus of the GRAVITY instrument [77] that has been installed at the Very Large Telescope Interferometer (VLTI) and operates at wavelengths of 2-2.4 microns. The detection of frame-dragging requires the discovery and subsequent monitoring of stars that are closer to the SMBH than those currently known [78], currently a difficult task due to the close proximity of the very bright star known as S2. Due to the elliptical nature of its orbit, this star will move away from the SMBH over the course of the next couple of years thereby allowing us access to these fainter stars that hold the key to the SMBH's spin.

\section{Astrophysical implications}

Measurements of black hole spin are providing new insights into the formation and growth of black holes. As already noted, X-ray reflection measurements of accreting SMBHs hint at two populations; a rapidly spinning $(a \gtrsim 0.9)$ population of black holes that dominate below approximately 30 million solar masses, and a more massive population of moderately spinning $(a \sim 0.4-0.7)$ SMBHs 33 . The very existence of a population of high-spin SMBHs is interesting, suggesting a significant population of SMBHs grew and were spun up by coherent disk accretion. The hint that lower-spin objects have higher-mass fits well with the hypothesis that today's high-mass SMBHs were built from more isotropic chaotic accretion or the merger of smaller black holes [5, 79, 80]. Implementing these ideas into future computer models of cosmological galaxy formation will allow predictions for the spin distribution of the SMBH population as a function of galaxy mass/type and time since the big bang.

Moving to the case of stellar-mass black holes, the spin is an indirect probe of the stellar corecollapse that created the black hole. The X-ray measurement of moderate- and high-spins in accreting stellar mass black holes stand in contrast to the very low spin rates inferred for newly created neutron stars, demonstrating clear differences in the physics of the supernovae that create these two classes of objects [81. A leading possibility is that neutron stars experience very strong magnetic braking during or shortly after their formation; if so, the transfer of energy from the rotation of the neutron star to the rest of the (exploding) star will be an important component of the supernovae mechanism.

With even just a small number of detections, gravitational wave measurements of low effective spin have relevance to the question of how these binary black holes may form. The most obvious route - a binary consisting of two regular massive stars that each evolve and collapse to black holes - is problematic. The tides of one star on the other would cause them to lock into synchronous rotation (i.e. the stars would be rotating with the same period as the orbit), resulting in rapidly spinning black holes aligned with the orbit once the stars collapse [82. This is incompatible with observed low 
effective spins. Possible solutions invoke binary systems consisting of unusual Wolf-Rayet stars which are too compact to lock into synchronous rotation, binary black holes that date back from the very first generation of star formed in the Universe, or black hole binary formation via dynamical capture processes in dense environments such as globular clusters or galactic nuclei [25, 83].

Current spin measurements are also providing insights into the physics of relativistic jet production. Rapidly spinning SMBHs are found in AGN both with and without powerful jets, proving that black hole spin is not by itself a sufficient ingredient for jet production (although it may still be a necessary ingredient). This is not in conflict with the idea that jets are powered by the magnetic extraction of black hole spin energy [3], instead suggesting that the strength and geometry of the magnetic field dictates whether strong jets are produced. This magnetic field strength is likely to depend on the state (temperature and geometric thickness) of the accretion disk, and hence the accretion rate, raising the possibility that AGN jets may turn on or off over long timescales. Such a picture is supported by studies of stellar-mass black holes where it is found that jets turn on when the accretion rate drops and the accretion disk becomes tenuous, hot, and geometrically thick. Turning this around, it may be possible to estimate spin in strongly jetted sources from measurement of jet power if we assume that the Blandford-Znajek mechanism is at work. Estimates of jet power from radio observations together with models for the jet launching based on the Blandford and Znajek mechanism have been used to estimate spin in powerful radio-galaxies where X-ray reflection signatures are weak and hard to measure [84, 85, 86].

\section{The future}

The coming decades will be tremendously exciting for black hole astrophysicists. The next generation of X-ray observatories, especially the future flagship mission ATHENA (the Advanced Telescopy for High-ENergy Astrophysics) that is approved and currently under development by the European Space Agency will allow further refinement of X-ray reflection and thermal continuum techniques, providing the sensitivity to study AGN at cosmological distances when the Universe was appreciably younger. The ability to measure the spin distribution of the SMBH population as the Universe ages will strongly constrain our models for how SMBHs first formed and then grew into today's monsters.

There are also a number of other X-ray techniques that have been explored for assessing spin in accreting black holes which have tremendous future potential. The gravitational microlensing of X-rays from multiply lensed quasars have already been used for gravitational redshift-based measures of spin in objects that are too distant and faint for more traditional X-ray reflection based techniques [87], and will be a focus of two NASA missions concepts currently under study, the probe-class Advanced X-ray Imaging Satellite (AXIS) and the flagship-class LYNX mission. Also, detailed timing of the variable $\mathrm{X}$-ray signal can in principal measure spin, both through the detection of X-ray echoes close to the black hole [88, 89, 90, 91, 92 and the characterisation of the quasi-periodic oscillations sometimes seen from black holes [93, 94, 95, 96. Probing the X-ray echoes will be a major theme of ATHENA and, as well as giving spin information, allows the structure of the inner accretion disk to be mapped. The theoretical interpretation of the quasi-periodic oscillations is still debated, but at least some of them may be due to spin-induced precession of the inner accretion disk [97, 95, 96] making them a potent diagnostic of black hole rotation with ATHENA as well as the Spectroscopic Time-Resolving Observatory for Broadband Energy X-rays (STROBE-X) mission which is currently under study by NASA.

The near future of gravitational wave astronomy will be characterized by improved sensitivity to merging stellar-mass objects together with improved ability to localize the source on the sky, enabling follow-up electromagnetic observations. Longer term, however, we must dramatically expand the range of frequencies that can be seen. The current generation of gravitational wave detectors cannot 
study binary SMBHs at all due to the restricted range of frequencies over which they can operate (approximately 10-1000 Hertz). The characteristic frequency of the gravitational waves is inversely proportional to the mass of the black holes, and so SMBH binaries have frequencies of milli-Hertz or less, far too low for aLIGO/VIRGO.

The overall background of very low-frequency gravitational waves (frequencies of approximately one cycle per year, or 30 nano-Hertz) from a Universe of SMBH binaries may be first detected through a very clever route - the NANOGrav project [98] is using radio telescopes to obtain exquisite timing of a large number of pulsars (spinning neutron stars) across the sky, seeking correlated disturbances to their apparent time keeping as a gravitational wave sweeps over Earth.

However, the detailed study of gravitational waves from SMBH binaries, including their spin, must wait for a space-based gravitational wave observatory such as the Laser Interferometer Space Antenna (LISA) which is approved and currently under development by ESA. LISA will be immune from the Earth-bound noise that limits today's ground-based detectors. Merging SMBHs emit unimaginable amounts of energy - during the actual merger event, they are by far the most energetic process in the entire Universe and can release the binding energy of an entire galaxy in a few minutes. This power will translate into high signal-to-noise detections of the gravitational wave train and precision measures of masses and spins. We must be aware of astrophysical selection effects, though. SMBHs that find themselves in binary systems and about to merge have undergone a very special history and the very process which brings the two black holes together in the first place may spin them up and/or align them [99]. To gain a full picture of the spins of the SMBH population, we need both gravitational wave probes of binaries, and electromagnetic (X-ray) probes of normal accreting SMBHs.

Finally, the ultimate, high-precision measurement of spin will be possible from the Galactic Centre black hole if only we discovered a radio pulsar close to the black hole. Pulsars are extremely accurate astrophysical clocks, and the timing of that clock in orbit around the black hole would allow its orbit to be constructed with superb accuracy and precision, determining the spin parameter to $0.1 \%$ [100]. However, we have yet to discovery a suitable pulsar close to the black hole. We would expect such pulsars to exist, so the most likely cause for non-detection is the phenomenon of "dispersion" by which the observed pulses are smeared out due to passage through ionized gas in the Galactic Centre. Dispersion can be corrected and the pulses de-smeared if we have a very sensitive radio observatory with excellent frequency resolution. We expect to detect a number of such pulsars with the Square Kilometer Array (SKA [101]), transforming the precision to which we know the spin of the Galactic Centre black hole.

The author declares no competing financial interests.

Please send correspondence to csr12@ast.cam.ac.uk. 


\section{References}

[1] Israel, W. Event Horizons in Static Vacuum Space-Times. Physical Review 164, 1776-1779 (1967).

[2] Carter, B. Axisymmetric Black Hole Has Only Two Degrees of Freedom. Physical Review Letters 26, 331-333 (1971).

[3] Blandford, R. D. \& Znajek, R. L. Electromagnetic extraction of energy from Kerr black holes. Mon. Not. R. astr. Soc. 179, 433-456 (1977).

[4] Fabian, A. C. Observational Evidence of Active Galactic Nuclei Feedback. Ann. Rev. Astr. Astrophys. 50, 455-489 (2012).

[5] Volonteri, M., Madau, P., Quataert, E. \& Rees, M. J. The Distribution and Cosmic Evolution of Massive Black Hole Spins. Astrophys. J. 620, 69-77 (2005).

[6] Miller, M. C. \& Miller, J. M. The masses and spins of neutron stars and stellar-mass black holes. Physics Reports 548, 1-34 (2015).

[7] Kerr, R. P. Gravitational Field of a Spinning Mass as an Example of Algebraically Special Metrics. Physical Review Letters 11, 237-238 (1963).

[8] Penrose, R. \& Floyd, R. M. Extraction of Rotational Energy from a Black Hole. Nature Physical Science 229, 177-179 (1971).

[9] Bambi, C. Spinning super-massive objects in galactic nuclei up to a $a_{\text {ast }}>1$. EPL (Europhysics Letters) 94, 50002 (2011).

[10] Bardeen, J. M., Press, W. H. \& Teukolsky, S. A. Rotating Black Holes: Locally Nonrotating Frames, Energy Extraction, and Scalar Synchrotron Radiation. Astrophys. J. 178, 347-370 (1972).

[11] Shakura, N. I. \& Sunyaev, R. A. Black holes in binary systems. Observational appearance. Astr. Astrophys. 24, 337-355 (1973).

[12] Reynolds, C. S. \& Fabian, A. C. Broad Iron-K $\alpha$ Emission Lines as a Diagnostic of Black Hole Spin. Astrophys. J. 675, 1048-1056 (2008).

[13] Shafee, R. et al. Three-Dimensional Simulations of Magnetized Thin Accretion Disks around Black Holes: Stress in the Plunging Region. Astrophys. J. Lett. 687, L25 (2008).

[14] Fabian, A. C. et al. Properties of AGN coronae in the NuSTAR era. Mon. Not. R. astr. Soc. 451, 4375-4383 (2015).

[15] George, I. M. \& Fabian, A. C. X-ray reflection from cold matter in active galactic nuclei and X-ray binaries. Mon. Not. R. astr. Soc. 249, 352-367 (1991).

[16] Fabian, A. C., Rees, M. J., Stella, L. \& White, N. E. X-ray fluorescence from the inner disc in Cygnus X-1. Mon. Not. R. astr. Soc. 238, 729-736 (1989).

[17] Laor, A. Line profiles from a disk around a rotating black hole. Astrophys. J. 376, 90-94 (1991).

[18] Brenneman, L. W. \& Reynolds, C. S. Constraining Black Hole Spin via X-Ray Spectroscopy. Astrophys. J. 652, 1028-1043 (2006). 
[19] Reynolds, C. S. et al. A Monte Carlo Markov Chain Based Investigation of Black Hole Spin in the Active Galaxy NGC 3783. Astrophys. J. 755, 88 (2012).

[20] Miller, L., Turner, T. J. \& Reeves, J. N. The absorption-dominated model for the X-ray spectra of typeI active galaxies: MCG-6-30-15. Mon. Not. R. astr. Soc. 399, L69-L73 (2009).

[21] Reynolds, C. S. Constraints on Compton-thick Winds from Black Hole Accretion Disks: Can We See the Inner Disk? Astrophys. J. Lett. 759, L15 (2012).

[22] Risaliti, G. et al. A rapidly spinning supermassive black hole at the centre of NGC 1365. Nature 494, 449-451 (2013).

[23] Taylor, C. \& Reynolds, C. S. Exploring the Effects of Disk Thickness on the Black Hole Reflection Spectrum. Astrophys. J. 855, 120 (2018).

[24] Tanaka, Y. et al. Gravitationally redshifted emission implying an accretion disk and massive black hole in the active galaxy MCG-6-30-15. Nature 375, 659-661 (1995).

[25] Miller, J. M. et al. Evidence of Spin and Energy Extraction in a Galactic Black Hole Candidate: The XMM-Newton/EPIC-pn Spectrum of XTE J1650-500. Astrophys. J. Lett. 570, L69-L73 (2002).

[26] Reynolds, C. S. \& Nowak, M. A. Fluorescent iron lines as a probe of astrophysical black hole systems. Physics Reports 377, 389-466 (2003).

[27] Miller, J. M. Relativistic X-Ray Lines from the Inner Accretion Disks Around Black Holes. Ann. Rev. Astr. Astrophys. 45, 441-479 (2007).

[28] Walton, D. J., Nardini, E., Fabian, A. C., Gallo, L. C. \& Reis, R. C. Suzaku observations of 'bare' active galactic nuclei. Mon. Not. R. astr. Soc. 428, 2901-2920 (2013).

[29] Xu, Y. et al. Evidence for Relativistic Disk Reflection in the Seyfert 1h Galaxy/ULIRG IRAS 05189-2524 Observed by NuSTAR and XMM-Newton. Astrophys. J. 837, 21 (2017).

[30] Ghosh, R., Dewangan, G. C., Mallick, L. \& Raychaudhuri, B. Broad-band spectral study of the jet-disc emission in the radio-loud narrow-line Seyfert 1 galaxy $1 \mathrm{H} 0323+342$. Mon. Not. R. astr. Soc. 479, 2464-2475 (2018).

[31] Walton, D. J. et al. Disentangling the complex broad-band X-ray spectrum of IRAS 13197-1627 with NuSTAR, XMM-Newton and Suzaku. Mon. Not. R. astr. Soc. 473, 4377-4391 (2018).

[32] Sun, S. et al. Multi-epoch analysis of the X-ray spectrum of the active galactic nucleus in NGC 5506. Mon. Not. R. astr. Soc. 478, 1900-1910 (2018).

[33] Reynolds, C. S. Measuring Black Hole Spin Using X-Ray Reflection Spectroscopy. Space Sci. Rev. 183, 277-294 (2014).

[34] Vasudevan, R. V. et al. A selection effect boosting the contribution from rapidly spinning black holes to the cosmic X-ray background. Mon. Not. R. astr. Soc. 458, 2012-2023 (2016).

[35] Brenneman, L. W. et al. The Spin of the Supermassive Black Hole in NGC 3783. Astrophys. J. 736, 103 (2011).

[36] Gandhi, P. et al. Gaia DR2 Distances and Peculiar Velocities for Galactic Black Hole Transients. ArXiv e-prints (2018). 
[37] Miller, J. M., Reynolds, C. S., Fabian, A. C., Miniutti, G. \& Gallo, L. C. Stellar-Mass Black Hole Spin Constraints from Disk Reflection and Continuum Modeling. Astrophys. J. 697, 900-912 (2009).

[38] Novikov, I. D. \& Thorne, K. S. Astrophysics of black holes. In Dewitt, C. \& Dewitt, B. S. (eds.) Black Holes (Les Astres Occlus), 343-450 (1973).

[39] Zhang, S. N., Cui, W. \& Chen, W. Black Hole Spin in X-Ray Binaries: Observational Consequences. Astrophys. J. Lett. 482, L155-L158 (1997).

[40] Davis, S. W. \& Hubeny, I. A Grid of Relativistic, Non-LTE Accretion Disk Models for Spectral Fitting of Black Hole Binaries. Astrophys. J. Suppl. 164, 530-535 (2006).

[41] Orosz, J. A. et al. Dynamical Evidence for a Black Hole in the Microquasar XTE J1550-564. Astrophys. J. 568, 845-861 (2002).

[42] Remillard, R. A. \& McClintock, J. E. X-Ray Properties of Black-Hole Binaries. Ann. Rev. Astr. Astrophys. 44, 49-92 (2006).

[43] Shafee, R. et al. Estimating the Spin of Stellar-Mass Black Holes by Spectral Fitting of the X-Ray Continuum. Astrophys. J. Lett. 636, L113-L116 (2006).

[44] McClintock, J. E. et al. The Spin of the Near-Extreme Kerr Black Hole GRS 1915+105. Astrophys. J. 652, 518-539 (2006).

[45] Gou, L. et al. The Extreme Spin of the Black Hole in Cygnus X-1. Astrophys. J. 742, 85 (2011).

[46] McClintock, J. E., Narayan, R. \& Steiner, J. F. Black Hole Spin via Continuum Fitting and the Role of Spin in Powering Transient Jets. Space Sci. Rev. 183, 295-322 (2014).

[47] Hubeny, I., Blaes, O., Krolik, J. H. \& Agol, E. Non-LTE Models and Theoretical Spectra of Accretion Disks in Active Galactic Nuclei. IV. Effects of Compton Scattering and Metal Opacities. Astrophys. J. 559, 680-702 (2001).

[48] Lawrence, A. Quasar viscosity crisis. Nature Astronomy 2, 102-103 (2018).

[49] Czerny, B., Hryniewicz, K., Nikołajuk, M. \& Sądowski, A. Constraints on the black hole spin in the quasar SDSS J094533.99+100950.1. Mon. Not. R. astr. Soc. 415, 2942-2952 (2011).

[50] Done, C., Jin, C., Middleton, M. \& Ward, M. A new way to measure supermassive black hole spin in accretion disc-dominated active galaxies. Mon. Not. R. astr. Soc. 434, 1955-1963 (2013).

[51] Capellupo, D. M., Wafflard-Fernandez, G. \& Haggard, D. A Comparison of Two Methods for Estimating Black Hole Spin in Active Galactic Nuclei. Astrophys. J. Lett. 836, L8 (2017).

[52] Piotrovich, M. Y., Gnedin, Y. N., Natsvlishvili, T. M. \& Buliga, S. D. Constraints on spin of a supermassive black hole in quasars with big blue bump. Astrophys. Space Sci. 362, 231 (2017).

[53] Abbott, B. P. et al. Observation of Gravitational Waves from a Binary Black Hole Merger. Physical Review Letters 116, 061102 (2016).

[54] Ajith, P. et al. Inspiral-Merger-Ringdown Waveforms for Black-Hole Binaries with Nonprecessing Spins. Physical Review Letters 106, 241101 (2011). 
[55] Schmidt, P., Ohme, F. \& Hannam, M. Towards models of gravitational waveforms from generic binaries: II. Modelling precession effects with a single effective precession parameter. Phys. Rev. D1 91, 024043 (2015).

[56] Abbott, B. P. et al. GW151226: Observation of Gravitational Waves from a 22-Solar-Mass Binary Black Hole Coalescence. Physical Review Letters 116, 241103 (2016).

[57] Abbott, B. P. et al. GW170104: Observation of a 50-Solar-Mass Binary Black Hole Coalescence at Redshift 0.2. Physical Review Letters 118, 221101 (2017).

[58] Abbott, B. P. et al. GW170608: Observation of a 19 Solar-mass Binary Black Hole Coalescence. Astrophys. J. Lett. 851, L35 (2017).

[59] Abbott, B. P. et al. Improved Analysis of GW150914 Using a Fully Spin-Precessing Waveform Model. Physical Review X 6, 041014 (2016).

[60] Detweiler, S. Black holes and gravitational waves. III - The resonant frequencies of rotating holes. Astrophys. J. 239, 292-295 (1980).

[61] Baibhav, V., Berti, E., Cardoso, V. \& Khanna, G. Black hole spectroscopy: Systematic errors and ringdown energy estimates. Phys. Rev. D1 97, 044048 (2018).

[62] Rees, M. J., Begelman, M. C., Blandford, R. D. \& Phinney, E. S. Ion-supported tori and the origin of radio jets. Nature 295, 17-21 (1982).

[63] Narayan, R., Yi, I. \& Mahadevan, R. Explaining the spectrum of Sagittarius A* with a model of an accreting black hole. Nature 374, 623-625 (1995).

[64] Falcke, H. \& Markoff, S. B. Toward the event horizon - the supermassive black hole in the Galactic Center. Classical and Quantum Gravity 30, 244003 (2013).

[65] Goddi, C. et al. BlackHoleCam: Fundamental physics of the galactic center. International Journal of Modern Physics D 26, 1730001-239 (2017).

[66] Falcke, H., Melia, F. \& Agol, E. Viewing the Shadow of the Black Hole at the Galactic Center. Astrophys. J. Lett. 528, L13-L16 (2000).

[67] Doeleman, S. S. et al. Event-horizon-scale structure in the supermassive black hole candidate at the Galactic Centre. Nature 455, 78-80 (2008).

[68] Fish, V. L. et al. $1.3 \mathrm{~mm}$ Wavelength VLBI of Sagittarius A*: Detection of Time-variable Emission on Event Horizon Scales. Astrophys. J. Lett. 727, L36 (2011).

[69] Gralla, S. E., Lupsasca, A. \& Strominger, A. Observational signature of high spin at the Event Horizon Telescope. Mon. Not. R. astr. Soc. 475, 3829-3853 (2018).

[70] Broderick, A. E., Fish, V. L., Doeleman, S. S. \& Loeb, A. Evidence for Low Black Hole Spin and Physically Motivated Accretion Models from Millimeter-VLBI Observations of Sagittarius A*. Astrophys. J. 735, 110 (2011).

[71] Broderick, A. E. et al. Modeling Seven Years of Event Horizon Telescope Observations with Radiatively Inefficient Accretion Flow Models. Astrophys. J. 820, 137 (2016).

[72] Medeiros, L. et al. GRMHD Simulations of Visibility Amplitude Variability for Event Horizon Telescope Images of Sgr A*. Astrophys. J. 856, 163 (2018). 
[73] Schödel, R. et al. A star in a 15.2-year orbit around the supermassive black hole at the centre of the Milky Way. Nature 419, 694-696 (2002).

[74] Ghez, A. M. et al. Stellar Orbits around the Galactic Center Black Hole. Astrophys. J. 620, 744-757 (2005).

[75] Ghez, A. M. et al. Measuring Distance and Properties of the Milky Way's Central Supermassive Black Hole with Stellar Orbits. Astrophys. J. 689, 1044-1062 (2008).

[76] Waisberg, I. et al. What stellar orbit is needed to measure the spin of the Galactic centre black hole from astrometric data? Mon. Not. R. astr. Soc. 476, 3600-3610 (2018).

[77] Eisenhauer, F. et al. GRAVITY: getting to the event horizon of Sgr A*. In Optical and Infrared Interferometry, vol. 7013 of Proc. of the SPIE, 70132A (2008).

[78] Gravity Collaboration et al. Detection of the gravitational redshift in the orbit of the star S2 near the Galactic centre massive black hole. Astr. Astrophys. 615, L15 (2018).

[79] Sesana, A., Barausse, E., Dotti, M. \& Rossi, E. M. Linking the Spin Evolution of Massive Black Holes to Galaxy Kinematics. Astrophys. J. 794, 104 (2014).

[80] Fiacconi, D., Sijacki, D. \& Pringle, J. E. Galactic nuclei evolution with spinning black holes: method and implementation. Mon. Not. R. astr. Soc. (2018).

[81] Miller, J. M., Miller, M. C. \& Reynolds, C. S. The Angular Momenta of Neutron Stars and Black Holes as a Window on Supernovae. Astrophys. J. Lett. 731, L5 (2011).

[82] Hotokezaka, K. \& Piran, T. Implications of the Low Binary Black Hole Aligned Spins Observed by LIGO. Astrophys. J. 842, 111 (2017).

[83] Stone, N. C., Küpper, A. H. W. \& Ostriker, J. P. Formation of massive black holes in galactic nuclei: runaway tidal encounters. Mon. Not. R. astr. Soc. 467, 4180-4199 (2017).

[84] Daly, R. A. Estimates of black hole spin properties of 55 sources. Mon. Not. R. astr. Soc. 414, 1253-1262 (2011).

[85] Daly, R. A. \& Sprinkle, T. B. Black hole spin properties of 130 AGN. Mon. Not. R. astr. Soc. 438, 3233-3242 (2014).

[86] Mikhailov, A. G. \& Gnedin, Y. N. Determination of the Spins of Supermassive Black Holes in FR I and FR II Radio Galaxies. Astronomy Reports 62, 1-8 (2018).

[87] Chartas, G. et al. Measuring the Innermost Stable Circular Orbits of Supermassive Black Holes. Astrophys. J. 837, 26 (2017).

[88] Reynolds, C. S., Young, A. J., Begelman, M. C. \& Fabian, A. C. X-Ray Iron Line Reverberation from Black Hole Accretion Disks. Astrophys. J. 514, 164-179 (1999).

[89] Fabian, A. C. et al. Broad line emission from iron K- and L-shell transitions in the active galaxy 1H0707-495. Nature 459, 540-542 (2009).

[90] Zoghbi, A., Fabian, A. C., Reynolds, C. S. \& Cackett, E. M. Relativistic iron K X-ray reverberation in NGC 4151. Mon. Not. R. astr. Soc. 422, 129-134 (2012). 
[91] Kara, E. et al. Discovery of high-frequency iron K lags in Ark 564 and Mrk 335. Mon. Not. R. astr. Soc. 434, 1129-1137 (2013).

[92] Cackett, E. M. et al. Modelling the broad Fe K $\alpha$ reverberation in the AGN NGC 4151. Mon. Not. R. astr. Soc. 438, 2980-2994 (2014).

[93] Cui, W., Zhang, S. N. \& Chen, W. Evidence for Frame Dragging around Spinning Black Holes in X-Ray Binaries. Astrophys. J. Lett. 492, L53-L57 (1998).

[94] Abramowicz, M. A. \& Kluźniak, W. A precise determination of black hole spin in GRO J1655-40. Astr. Astrophys. 374, L19-L20 (2001).

[95] Ingram, A., Done, C. \& Fragile, P. C. Low-frequency quasi-periodic oscillations spectra and Lense-Thirring precession. Mon. Not. R. astr. Soc. 397, L101-L105 (2009).

[96] Motta, S. E. et al. Black hole spin measurements through the relativistic precession model: XTE J1550-564. Mon. Not. R. astr. Soc. 439, L65-L69 (2014).

[97] Fragile, P. C., Blaes, O. M., Anninos, P. \& Salmonson, J. D. Global General Relativistic Magnetohydrodynamic Simulation of a Tilted Black Hole Accretion Disk. Astrophys. J. 668, 417-429 (2007).

[98] Arzoumanian, Z. et al. The NANOGrav Nine-year Data Set: Limits on the Isotropic Stochastic Gravitational Wave Background. Astrophys. J. 821, 13 (2016).

[99] Bogdanović, T., Reynolds, C. S. \& Miller, M. C. Alignment of the Spins of Supermassive Black Holes Prior to Coalescence. Astrophys. J. Lett. 661, L147-L150 (2007).

[100] Liu, K., Wex, N., Kramer, M., Cordes, J. M. \& Lazio, T. J. W. Prospects for Probing the Spacetime of Sgr A* with Pulsars. Astrophys. J. 747, 1 (2012).

[101] Keane, E. et al. A Cosmic Census of Radio Pulsars with the SKA. Advancing Astrophysics with the Square Kilometre Array (AASKA14) 40 (2015). 